\title{
Microwave-Assisted Green Synthesis of Carbon Quantum Dots Derived from Calotropis Gigantea as a Fluorescent Probe for Bioimaging
}

\section{Neetu Sharma}

Maharishi Markandeshwar University

Indu Sharma

Maharishi Markandeshwar University

Milan Kumar Bera ( $\nabla$ m.k.bera@mmumullana.org )

Maharishi Markandeshwar University https://orcid.org/0000-0002-2996-890X

\section{Research Article}

Keywords: Green synthesis, Fluorescent, Carbon quantum dots, Fluorescent staining, Bioimaging

Posted Date: October 22nd, 2021

DOl: https://doi.org/10.21203/rs.3.rs-1000229/v1

License: (c) (i) This work is licensed under a Creative Commons Attribution 4.0 International License.

Read Full License

Version of Record: A version of this preprint was published at Journal of Fluorescence on March 9th, 2022. See the published version at https://doi.org/10.1007/s10895-022-02923-4. 


\title{
Microwave-Assisted Green Synthesis of Carbon Quantum Dots Derived from Calotropis Gigantea as a Fluorescent Probe for Bioimaging
}

\author{
Neetu Sharma ${ }^{1, a}$, Indu Sharma ${ }^{2, b}$ and Milan Kumar Bera ${ }^{1, c^{*}}$ \\ ${ }^{1}$ Department of Physics, Maharishi Markandeshwar (Deemed to be University), Mullana. Ambala \\ (133207), Haryana, India \\ ${ }^{2}$ Department of Biotechnology, Maharishi Markandeshwar (Deemed to be University), Mullana. \\ Ambala (133207), Haryana, India \\ \#corresponding e-mail: m.k.bera@mmumullana.org \\ ORCID ID: 0000-0002-2996-890X
}

\begin{abstract}
In this study, an eco-friendly, cost-effective, and convenient method for preparing biocompatible fluorescent carbon quantum dots (CQDs) by one-pot microwave assisted synthesis from the leaf extract of the medicinal plant Calotropis gigantea, also known as crown flower, has been demonstrated. As-synthesized CQDs demonstrated fluorescence quantum yields up to 4.24 percent. The size distribution of the as-synthesized CQDs varied from 2.7 to $10.4 \mathrm{~nm}$, with a significant proportion of $\mathrm{sp}^{2}$ and $\mathrm{sp}^{3}$ carbon groups verified by nuclear magnetic resonance analysis. The zeta potential of as-synthesized CQDs was measured to be $-13.8 \mathrm{mV}$, indicating the existence of a negatively charged surface with incipient instability in aqueous suspension. Furthermore, as an alternative to organic or synthetic dyes, the development of simple, inexpensive, and non-destructive fluorescence-based staining agents are highly desired. In this regard, as-synthesized CQDs shown remarkable fluorescent staining capabilities in this work and may be utilised as a suitable probe for optical and bio-imaging of bacteria, fungi, and plant cells.
\end{abstract}

Keywords: Green synthesis, Fluorescent, Carbon quantum dots, Fluorescent staining, Bioimaging. 


\section{Introduction}

Carbonaceous and carbon-based nanomaterials have received a great deal of interest in recent years because to their enormous potential for practical applications in photocatalysis, optoelectronics, biomedicines, thin film displays, drug delivery, and other engineering and medical disciplines [1-6]. Since their surprising discovery in 2004, carbon quantum dots (CQDs) have demonstrated outstanding photo stability, tiny size, highly regulated photoluminescence, biocompatibility, electrochemiluminescence, and extraordinary multi-photon excitation (up-conversion) capacity [711]. Since CQDs may be functionalized with biomolecules and are less toxic and chemically inert in nature, hence, they are suitable carriers for drug delivery, biological imaging, and other applications [12-17]. Semiconducting quantum dots are often synthesized using relatively expensive precursors, however, repeatability remains a challenge $[18,19]$. Hence, it is critical to develop and produce a simple, cost-effective, large-scale ecofriendly method/process that makes use of low-cost precursors. In this context, CQDs provide unique and critical features such as excellent biocompatibility, particular biological target, minimum toxicity, and a robust quantum size impact when compared to conventional inorganic QDs, which can be produced utilising low-cost methods [20-24].

In this senario, natural biomaterial resources are typically preferred over other organic, inorganic, or synthetic resources in the search for an efficient, inexpensive, and environmentally friendly synthesis method of CQDs because they are renewable and biocompatible, and can convert biomass waste into worthy and valuable materials [25]. Natural precursors are also potential chemical alternatives. Besides, natural precursors outperform chemicals in the synthesis of carbon-based nanomaterials due to a variety of advantages, including cheap cost, nontoxicity, and availability [26,27]. Several studies on the synthesis of carbon-based nanomaterials from natural precursors, including orange juice, green tea, egg, potatoes, lotus root, pepper, coriander leaves, and others, have recently been reported [28-34].

Green synthesis methods, on the other hand, are considerably more acceptable than physical and chemical procedures. In comparison to traditional time-consuming hydrothermal procedures, microwave-assisted bottom-up processes may be advantageous for rapid, easy one-pot synthesis, efficient, cost-effective, and energy-saving methods of synthesising high quantum yield carbon quantum dots.

In the meantime the fluorescent nanomaterials have sparked a lot of attention as potential competitors to traditional fluorescent dye probes in recent years, and they've evolved quickly as a result of the growing need for fluorescent probes in chemical sensing, biological labelling, and other sectors. When compared to ordinary fluorescent dyes, fluorescent nanomaterials have the quantum size effect and unique nanomaterial effects, which may overcome many of the latter's disadvantages, such as poor fluorescence intensity, low stability, quick photo bleaching, and so on. Fluorescent 
nanoparticles such as gold quantum dots, cupper or palladiu nanoclustersare currently widely employed in a variety of scientific and technical sectors [35-39].

One of the primary goals of this research is to develop a simple and efficient experimental method for low-cost CQD fabrication from the leaf extract of the traditional medicinal plant known as crown flower (Calotropis gigantea) using one-step microwave-assisted synthesis at relatively low temperatures and in a shorter time frame. Calotropis gigantea is a medicinal herb that has been used for thousands of years. Calotropis gigantea is a traditional medicinal plant native to Asian countries that belongs to the "Asclepiadaceous" family and is endowed with immense medicinal properties that are frequently used as Ayurvedic medicine to treat a variety of illnesses such as toothache, earache, sprain, anxiety, pain, epilepsy, diarrhoea, and so on [35].

Meanwhile, the use of fluorescent stains or markers to see prokaryotic (bacteria) and eukaryotic (fungi and plants) cells is rapidly spreading across all disciplines [36]. Microorganisms may be observed directly under a microscope, which is a useful tool in many microbiological studies. This has been seen with protozoa, fungi, injected bacteria, and rhizosphere microorganisms.

Fluorescent dyes have traditionally been used to stain members of many bacterial genera, fungi, and plant cells. Both cationic and anionic dyes (also known as fluorochromes if fluorescent) have been utilised because of their ability to attach to particular biological components of microbial cells. Fluorochromes that are widely employed include acridine orange, ethidium bromide, fluorescein isothiocyanate, and others [40]. However, for a sustainable future, the employment of biocompatible, non-toxic, ecologically friendly, and cost-effective fluorescent materials is important. As a consequence, in this study, we demonstrated the essential material and optical properties of CQDs synthesised from Calotropis gigantea leaf extract, as well as a fluorescent staining approach for utilising those CQDs for biological imaging and plant cell markers.

\section{Experimantal section \\ 2.1.Chemicals and Materials}

Fresh Calotropis gigantea (crown flower) leaves were collected in Bhambhol, Yamunanagar, Haryana, India. The chemicals used in this investigation were all of analytical grade. Other materials purchased from a commercial supplier included Whatman filter paper (grade 1), $0.22 \mu \mathrm{m}$ syringe filter (polytetrafluoroethylene membrane), acetone, and isopropyl alcohol. Fluorescent staining was done with Bacillus subtilis, Escherichia coli, bacteria Aspergillus fungi, and Tradescantia pathacea (Sitara Plant). A Milli-Q system was used to obtain deionized water (18.2 M $\Omega . \mathrm{cm}^{-1}$ ) (Millipore, France). 


\subsection{Microwave-assisted Green Synthesis of Carbon Quantum Dots (CQDs)}

Fresh Calotropis gigantea (crown flower) leaves were collected and processed for extraction. The leaves were thoroughly rinsed with tap water, then distilled water to remove dust particles, air-dried, and incised into small pieces. In the beginning, $10 \mathrm{gm}$ of fresh crown flower leaves were crushed into a powder in a clean environment and mixed with $100 \mathrm{ml}$ of distilled water. The blended aqueous extract solution was filtered through Whatman qualitative filter paper (grade 1) to remove fibrous impurities before being placed in a $900 \mathrm{~W}$ domestic microwave oven [41]. The microwave processing time was meticulously adjusted until brown viscus fluid was obtained. To remove the large agglomerate particles afterwards, it was centrifuged for 15 minutes at $15000 \mathrm{rpm}$. A $0.22-\mu \mathrm{m}$ syringe filter was then used to filter the supernatant (polytetrafluoroethylene membrane). Finally, the watersuspended CQD solution was kept at $4{ }^{\circ} \mathrm{C}$ for future use. The complete procedure is depicted graphically in Figure 1.

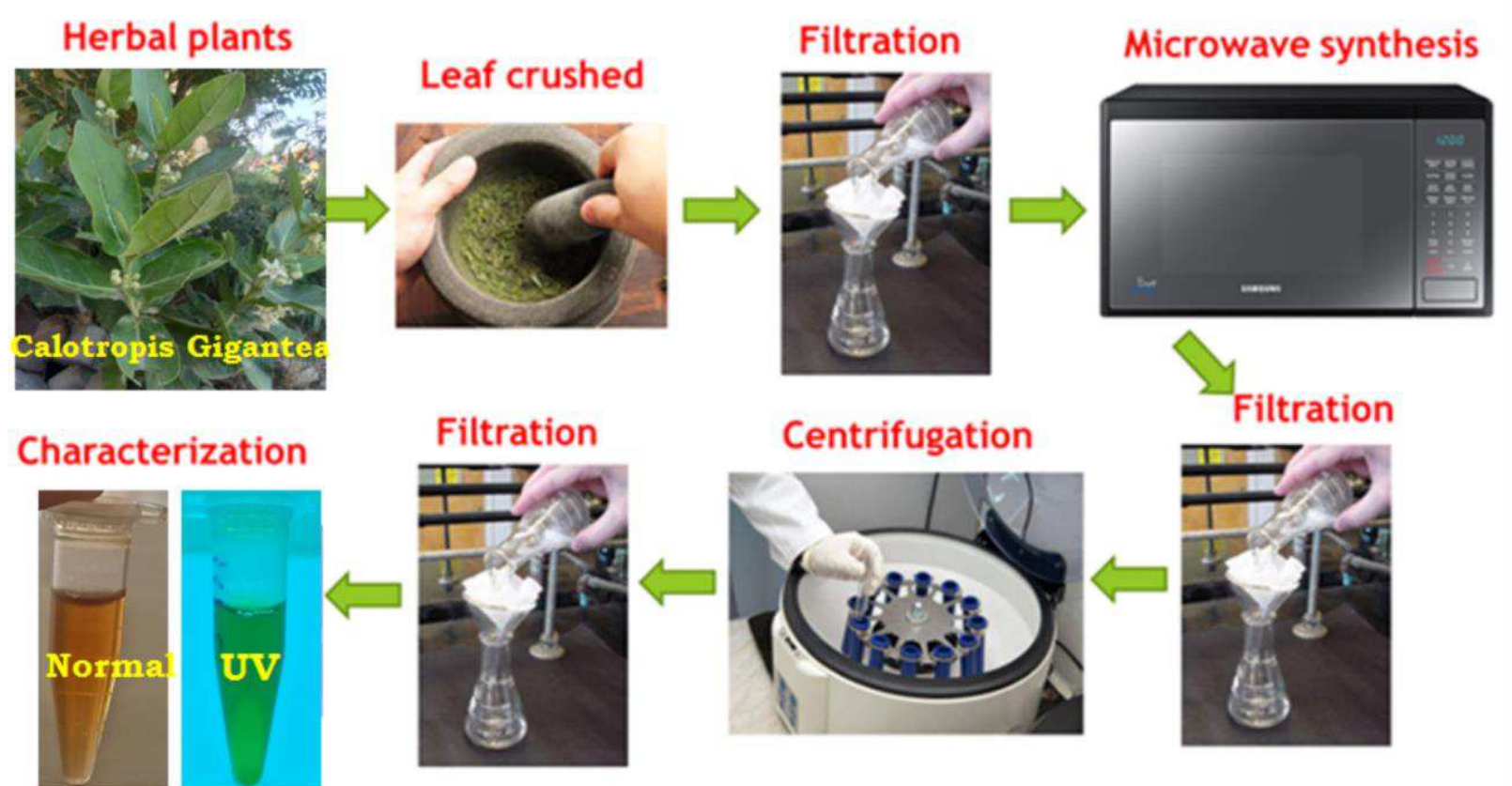

Figure 1. Schematic representation of the synthesis of CQDs from medicinal plant Calotropis Gigantea leaf extract through microwave-assisted process.

\subsection{Apparatus and Characterization}

The as-synthesized CQDs were characterised using several characterisation methods. Shimadzu UV-2700 spectrophotometer was used to measure UV-visible absorption spectra. Varian Cary Eclipse fluorescence spectrophotometer was used to record the fluorescence emission spectra. Transmission electron microscopy was used to confirm the average size and morphology of the CQDs (Hitachi, H7500). The TEM analysis yielded selected area electron diffraction (SAED). Panalytical's X'Pert Pro was used to generate the powder X-ray diffraction (XRD) pattern. The Fourier transform infrared spectrum (FTIR) was measured on a (PerkinElmer Spectrum 100) in the 500 to $4000 \mathrm{~cm}^{-1}$ range. The 
Nano Zeta-Sizer Malvern apparatus was used to determine the zeta potential. The nuclear magnetic resonance (NMR) spectroscopy (FT-NMR) was carried out using an FT-NMR spectrometer (Avance Neo, Bruker). Fluorescence images were captured using a Nikon Eclipse E600 fluorescence microscope.

\subsection{Quantum Yield Determination}

As a standard, conventional quinine sulphate $\left(0.1 \mathrm{M} \mathrm{H}_{2} \mathrm{SO}_{4}\right.$ as solvent; $\left.\mathrm{QY}=0.54\right)$ was chosen. The slope method was used to determine the QY of CQDs (in water) with reference to quinine sulphate. The curve was obtained by comparing the photoluminescence intensity and absorbance values of the samples to those of the reference (several values less than 0.1 at excitation wavelength). The equation used for QY calculation is [42]:

$$
Q Y_{Q D}=Q Y_{\text {ref }} \cdot \frac{I_{Q D}}{I_{\text {ref }}} \cdot \frac{A_{\text {ref }}}{A_{Q D}} \cdot \frac{\eta_{Q D}^{2}}{\eta_{r e f}^{2}}
$$

where QY ref is the quantum yield of the reference sample, $\eta$ is the refractive index of the solvent, $I$ is the integrated fluorescence intensity and $\mathrm{A}$ is the absorbance at the excitation wavelength. The CQDs were diluted in deionized water to achieve the necessary concentrations. A UV-Visible device was used to test the absorbance of CQDs at varied concentrations. The emission spectra of particular concentrations of CQDs were measured using a fluorescence spectrometer at an excitation light at $280 \mathrm{~nm}$, and the allied integrated intensity was computed. The QY of CQDs was found to be 4.24\%.

\subsection{Cell culture/ Staining Technique}

The gram-positive and gram-negative bacteria (Escherichia coli, Bacillus subtilis) were streaked on nutrient agar before being inoculated into liquid nutrient broth culture medium and grown at $37^{\circ} \mathrm{C}$ with 100 rpm shaking for 18-24 hours [43].

\subsection{Bacterial staining}

First, a bacterial smear was stained with fluorescent CQDs (concentration in the range between $\sim 0.1-0.2 \mathrm{mg} / \mathrm{ml}$ ) and allowed to dry before being heated. The smears were then held in place with a slide rack or a clothes pin. The fluorescent dye was then carefully applied to each smear. Before inspecting the strained slides under a fluorescent microscope, they were allowed to air dry.

\subsection{Fungal staining:}

To begin, a drop of fluorescent CQDs $(\sim 0.1-0.2 \mathrm{mg} / \mathrm{ml})$ was put on a clean slide, followed by a tiny tuft of fungal mycelium containing spores. Then, gently tease the material with the two mounted needles. Following that, the slide was viewed using a fluorescence microscope while covered with a cover slip to prevent air bubbles from being trapped in the stain. 


\subsection{Plant cell staining}

The removal of the leaf epidermis in order to examine the quantity, arrangement, distribution, and structure of stomata is known as peeling. The technique includes using force to shatter the leaf unevenly. This readily separates a little part of the bottom epidermis that is still protruding on the lower surface of the leaf. A lengthy ribbon or strip of lower epidermis is ripped off. Before being viewed under a fluorescent microscope, the lower epidermis is scraped, dyed with fluorescent CQDs $(\sim 0.1-0.2 \mathrm{mg} / \mathrm{ml})$, and coated with a coverslip. After that, the coverslip was put on the slide and inspected using a fluorescence microscope.

\subsection{Fluorescent stain screening:}

Bacterial growth was achieved using nutrient broth medium, which was incubated at room temperature for 24 hours $\left(30-37^{0} \mathrm{C}\right)$. On separate glass slides, tiny smears of microorganisms were produced first. Allow the smear mixture containing the produced fluorescent stain to dry naturally. Each smear was stained with fluorescent dye for 30 seconds. After that, the coverslip was put on the slide and inspected using a fluorescence microscope. When the adhesion values stabilised at 30 to $40 \%$, the fluorescent stains were screened using a biological fluorescent stain at an optimised concentration. Fluorescent stain at effective concentrations can be used to stain microorganisms at predictable places around and across contaminated regions, as determined by staining techniques. We concentrated on creating fluorescent staining techniques to identify and pinpoint the location of bacteria and fungus in diverse samples in this work.

\section{Results and discussion}

\subsection{Composition, structure and morphology analysis}

The size and morphology of as-synthesized CQDs derived from Calotropis gigantea were investigated using high resolution TEM. Figure 2a shows the almost spherical form of carbon dots, while Figure 2b shows the corresponding histogram, which indicates that the average size of CQDs is $5.7 \mathrm{~nm}$, with a range of 2.7 to $10.4 \mathrm{~nm}$. It is worth noting that CQDs are not evenly distributed; rather, they are attempting to agglomerate due to the absence of any passivating agent. In fact, the measured Zeta potential value was around $-13.8 \mathrm{mV}$, indicating the presence of a negatively charged surface with incipient instability in water suspension. Furthermore, the broad diffused ring in the selected area electron diffraction (SAED) pattern revealed the as-synthesized CQDs' poor polycrystalline nature (Fig. 2c). 

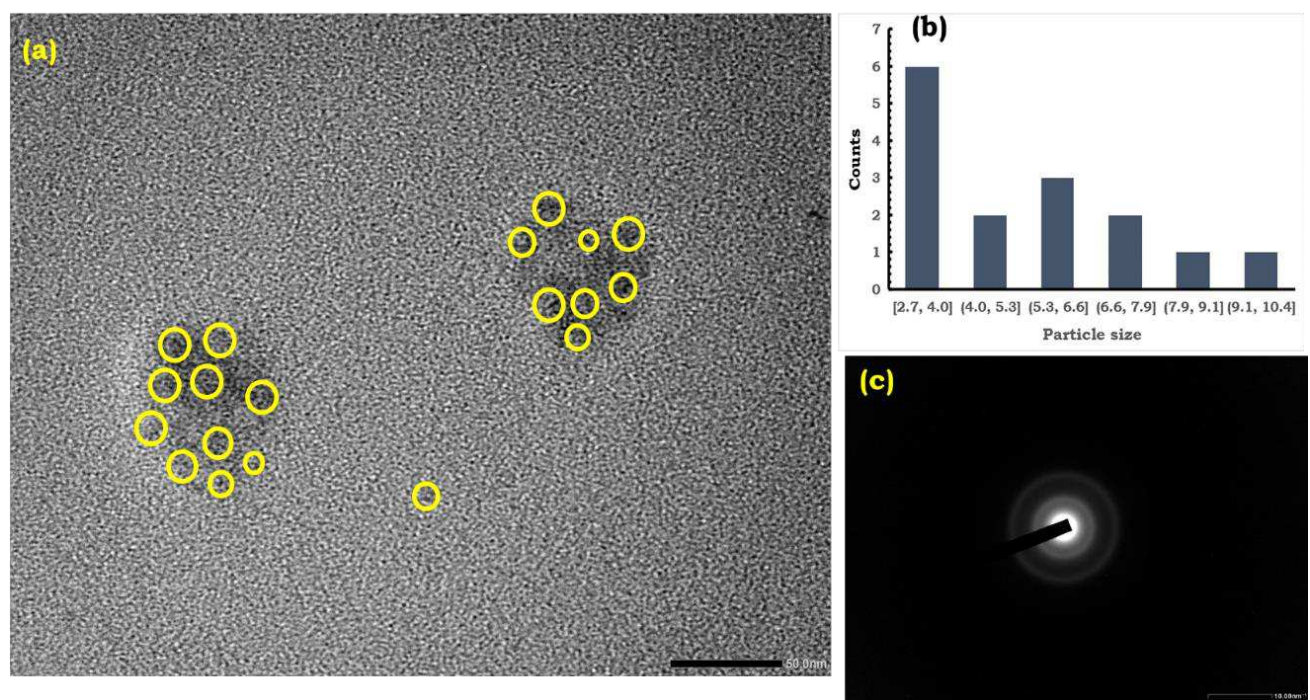

Figure 2. (a) High resolution TEM microimage of the synthesized CQDs, (b) size distribution graph and (c) SAED pattern.

XRD was used to investigate the crystalline nature of as-synthesized CQDs. X-ray diffraction pattern obtained with PANalytical X'pert Pro MPD powder X-ray diffractometer with $\mathrm{Cu} \mathrm{K} \alpha(\lambda=$ $1.54 \AA$ ) radiation confirmed relatively better crystalline nature. Figure 3's XRD spectrum confirms that the synthesized CQDs are poly-crystalline, with a broad peak around $2 \theta=25^{\circ}$ due to the presence of amorphous carbonaceous core-shell materials [44,45].

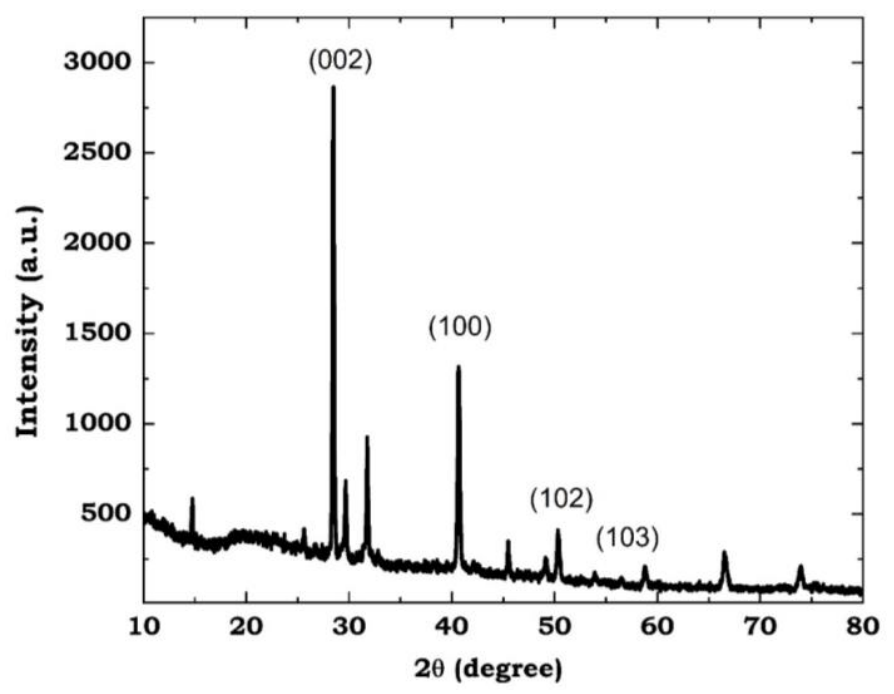

Figure 3. X-ray diffraction pattern of as synthesized carbon dots.

The observed relatively steep peaks at $28.4^{\circ}, 40.6^{\circ}, 50.3^{\circ}$, and $58.8^{\circ}$, however, correspond to the crystal planes (002), (100), (102), and (103), where the first three are graphite $\left(\mathrm{sp}^{2}\right)$ and the last one is diamond $\left(\mathrm{sp}^{3}\right)$, which is comparable to carbon [46]. The d-spacing for (002), (100), (102), and (103) planes was calculated to be $0.31,0.22,0.18$, and $0.16 \mathrm{~nm}$, respectively, which is similar to the graphitic lattice spacing [47]. 
On the other hand, FTIR data revealed the presence of various oxygen functional groups and linkages in carbon quantum dots (see Fig. 4). The presence of $-\mathrm{OH},-\mathrm{C}-\mathrm{H}, \mathrm{C}=\mathrm{O}, \mathrm{O}-\mathrm{H}$, and $\mathrm{C}-\mathrm{O}$ was clearly indicated by stretching frequencies at 3487, 2980,1745, 1380, and $1078 \mathrm{~cm}^{-1}$, respectively $[48,49]$. The presence of these functional groups suggests that the carbon quantum dots that were synthesized have a high water solubility.

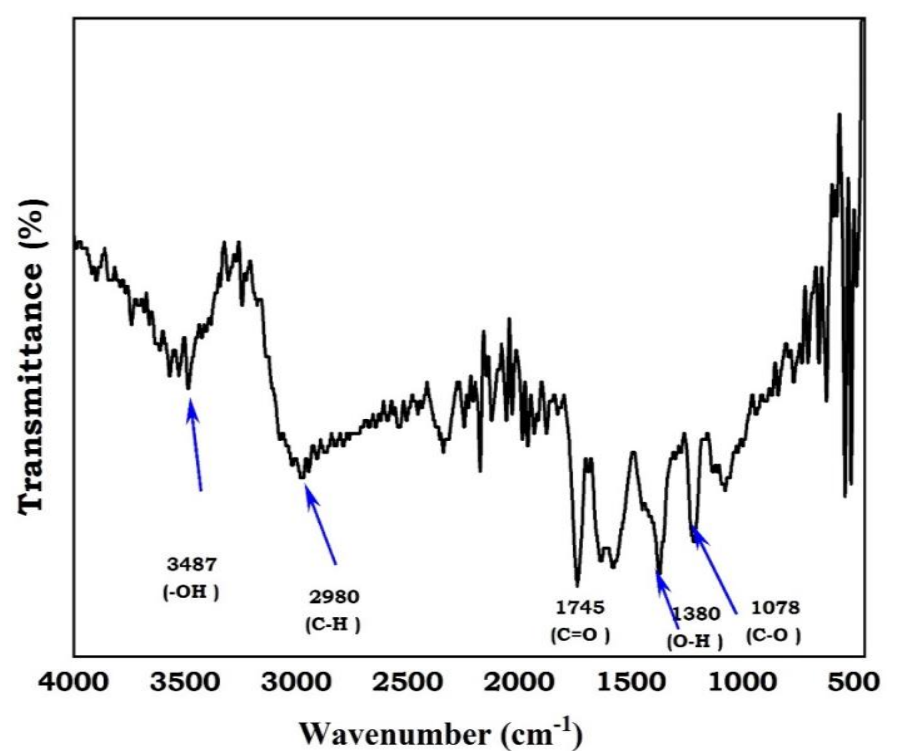

Figure 4. FT-IR spectrum of as synthesized carbon dots.

Furthermore, nuclear magnetic resonance (NMR) spectroscopy $\left({ }^{1} \mathrm{H}\right.$ and $\left.{ }^{13} \mathrm{C}\right)$ of CQDs was utilized to distinguish between $\mathrm{sp}^{3}$-hybridized carbon atoms and $\mathrm{sp}^{2}$-hybridized carbon atoms. The NMR spectra displayed in Fig. 5 demonstrate the presence of four different chemical environments in the four separate areas mentioned below.

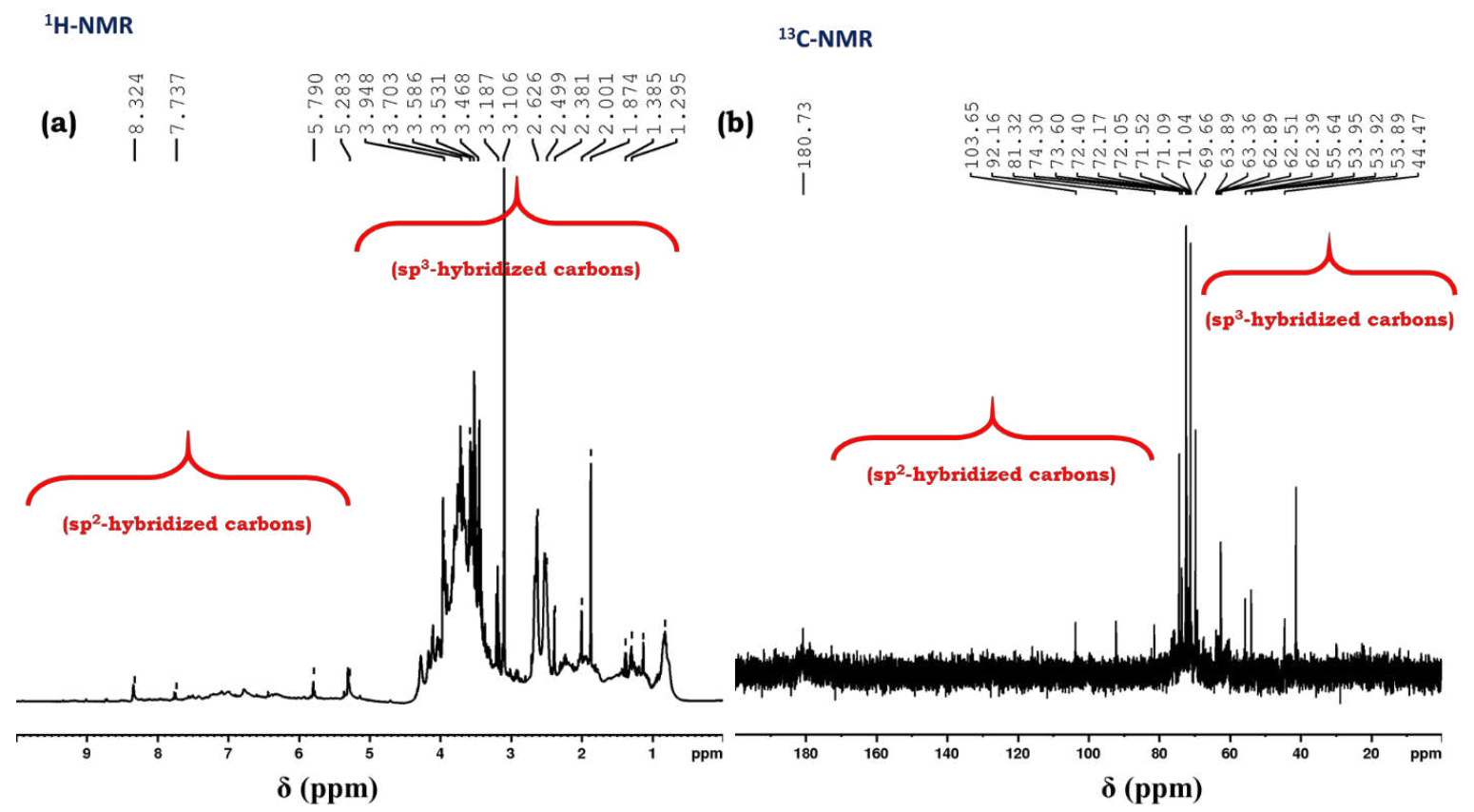

Figure 5. (a) ${ }^{1} \mathrm{H}-\mathrm{NMR}$ and (b) ${ }^{13} \mathrm{C}-\mathrm{NMR}$ spectra of the as-synthesized carbon dots. 
The following chemical shift ( $\delta$ ) regions were discovered in the ${ }^{1} \mathrm{H}$ NMR spectrum (Fig. 5a): 1-3 ppm (dominated by $\mathrm{sp}^{3} \mathrm{C}-\mathrm{H}$ protons), 3-6 ppm (associated with protons of hydroxyl, ether, and carbonyl groups), 6-8 ppm (for aromatic or $\mathrm{sp}^{2}$ protons), and 8-10 ppm (related to aldehydic protons) [50]. The ${ }^{1} \mathrm{H}$ NMR spectrum in Fig. 5a shows that the first two regions mentioned above are dominated over the last two regions, indicating that $\mathrm{sp}^{3}$-hybridized carbon atoms are dominated over $\mathrm{sp}^{2}$-hybridized carbon atoms. Carbon quantum dots are indeed mixed with $\mathrm{sp}^{3}$ and $\mathrm{sp}^{2}$-hybridized carbon atoms. Meanwhile, the ${ }^{13} \mathrm{C}$ NMR spectrum revealed a mixture of $\mathrm{sp}^{3}$ and $\mathrm{sp}^{2}$-hybridized carbon atoms (see Fig. 5b). There were four distinct regions found, namely 20-80 ppm (for $\mathrm{sp}^{3}$ carbons as well as carbons attached with hydroxyl groups), 80-100 ppm (for carbons attached with ether linkages), 100-120 ppm (for $\mathrm{C}=\mathrm{C}$ aromatic or $\mathrm{sp}^{2}$ hybridized carbon atoms), and finally 175190 ppm (for $\mathrm{C}=\mathrm{O}$ carbons) [51][51].

\subsection{Absorbance and luminescence properties}

The optical absorption peak of as-synthesized carbon dots was observed in the UV region, with a maximum absorption around $280 \mathrm{~nm}$ and a tail extending into the visible range (see Fig. 6 for detail). This is due to the usual $n-\pi^{*}$ transition of the $\mathrm{C}=\mathrm{O}$ band and the $\pi-\pi^{*}$ transition of the conjugated $\mathrm{C}=\mathrm{C}$ band [52]. Besides, UV-Visible spectrometry may also be used to display quantum or nano dots in order to measure their band gap energy and particle sizes. The cut-off wavelength was initially estimated by intersecting the peak's tangent line with the wavelength axis.

This wavelength is used to calculate the band gap $E_{g}^{C Q D}$ of the CQD [53]:

$$
E_{g}^{C Q D}=\frac{h c}{\lambda_{\text {edge }}}
$$

where $\lambda_{\text {edge }}=$ wavelength absorbed by the CQD sample, and $c$ is the speed of light. Firstly, a line of best fit was determined for the linear portion near the peak in the spectrum, as shown in Fig. 6. 


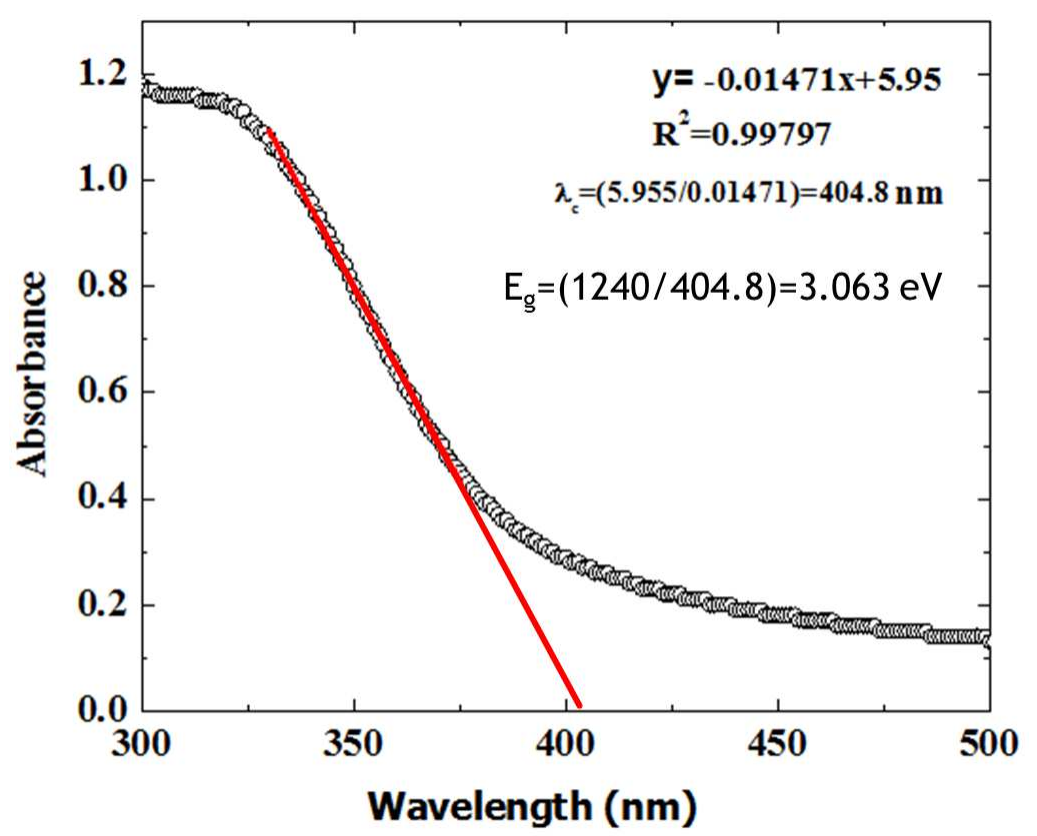

Figure 6. UV-vis absorption spectrum of as synthesized carbon dots from Calotropis gigantea.

The equation for best-fitting line is shown, along with an $\mathrm{R}^{2}$ value close to 1 to ensure that this portion is linear. Using this line's equation, the cut-off wavelength of $404.8 \mathrm{~nm}$ and band gap of $3.063 \mathrm{eV}$ were calculated. Secondly, using the following expression derived from the following effective mass model, the particle size can be estimated from the experimental UV-Vis absorption spectrum [53]:

$$
E_{g}^{C Q D}=E_{g}^{b u l k}+\frac{\hbar^{2} \pi^{2}}{2 r^{2}}\left(\frac{1}{m_{e}^{*}}+\frac{1}{m_{h}^{*}}\right)-\frac{1.8 e^{2}}{4 \pi \varepsilon \varepsilon_{0} r}-\frac{0.124 e^{4}}{\hbar^{2}\left(4 \pi \varepsilon \varepsilon_{0}\right)^{2}}\left(\frac{1}{m_{e}^{*}}+\frac{1}{m_{h}^{*}}\right)^{-1}
$$

where $E_{g}^{C Q D}=$ = band gap energy of CQD, which will be determined from the UV-Visible absorbance spectrum, $E_{g}^{\text {Bulk }}=$ band gap energy of the bulk at room temperature, $h=$ Planck's Constant, $6.625 \times 10^{-34} \mathrm{~J} \cdot \mathrm{s}, r=$ particle radius $(\mathrm{m}), m_{e}=$ mass of a free electron, $9.11 \times 10^{-31} \mathrm{~kg}, m_{e}^{*}$ $=0.19 m_{e}$ (effective mass of a conduction band electron), $m_{h}^{*}=0.80 m_{e}$ (effective mass of a valence band hole), $e=$ elementary charge, $1.602 \times 10^{-19} \mathrm{C}, \varepsilon_{0}=8.854 \times 10^{-12} \mathrm{C}^{2} \mathrm{~N}^{-1} \mathrm{~m}^{-2}$ (permittivity of free space), $\varepsilon$ is the relative permittivity. The estimated CQD size was around $3.82 \mathrm{~nm}$, which is consistent with the observed HRTEM images.

Meanwhile, carbon dots are distinguished by their emission wavelength and size-dependent fluorescent behavior. Figure 7 depicts the fluorescence spectrum of CQDs. The emission peak ranged from 340-480 nm after excitation with a wavelength of $280 \mathrm{~nm}$. The deconvoluted fluorescent spectrum revealed two distinct broad peaks, with maximum emission peaks occurring at 354 and 441 $\mathrm{nm}$, respectively, indicating multi-color fluorescent emission. 


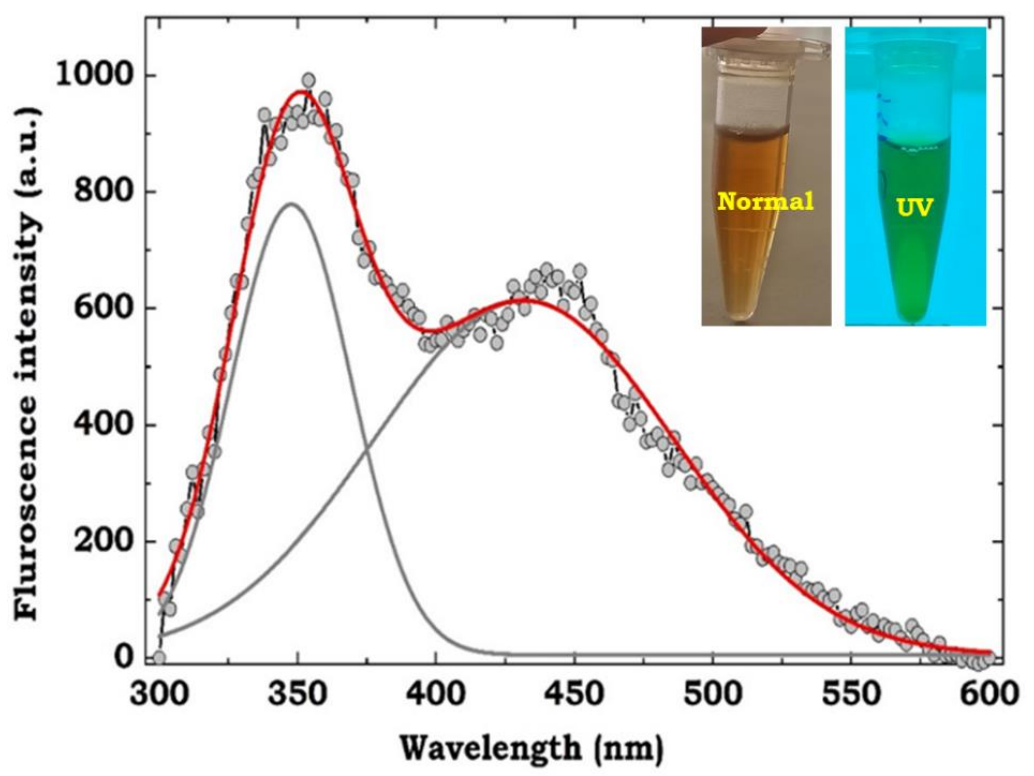

Figure 7. Fluorescence spectrum of as-synthesized carbon dots from Calotropis gigantea. Inset: Optical images of CQDs obtained under (a) visible light (the left vial) and (b) UV light $\left(\lambda_{\mathrm{ex}}=365\right.$ $\mathrm{nm}$ ) (the right vial).

\subsection{Development of a Fluorescent Staining Method for Monitoring Bacteria, Fungi and Plant cells}

The purpose of this research is to find alternative fluorescent stains or markers for bacteria, fungi, and plant cells. One of the most important conditions for any fluorescent stain is that it has very modest impacts on bacterial attachment, feasibility, and metabolic activity while remaining in cells for at least a few weeks in order to be utilised to investigate bacterial transport and monitor bioaugmentation. 5-sulfofluorescein diacetate, sodium salt (SFDA), 5-cyano-2,3-ditolyl-tetrazolium chloride (CTC), fluorescamine and other fluorescent staining agents have previously been reported in the literature [54]. During staining with such chemicals, the manufacturer's suggested methods, such as suitable carrier solvents, cell suspension buffers, final concentrations, and temperature settings, must all be followed. Besides, to achieve final concentrations, the compounds must be added as concentrated stocks in carrier solvents containing dimethyl sulfoxide (DMSO) or dimethyl formamide at the appropriate concentrations [54]. The aforementioned lengthier procedures, however, are not required for the new CQD-based fluorescent stain derived from Calotropis gigantea in this work. Despite the fact that the general staining technique was adequate for initial screening, an attempt was made to minimise the quantity of fluorescent stain needed to stain a specific number of cells while simultaneously optimising the overall staining procedure. This was selected primarily to reduce the expense of labelling cells for field-scale research while also allowing the staining procedure's scaling-up. Various staining combinations were performed such as staining of stationary cultures, staining of log-phase cultures, staining with ambient temperature $\left(32^{\circ} \mathrm{C}\right)$ or cycling temperature ranges from 25 to $37^{\circ} \mathrm{C}$ and staining with different fluorescent stain concentrations (10, 50, and $100 \mu \mathrm{l})$. Fluorescent staining typically took 2 to 4 hours. The staining was assessed 
qualitatively using a Nikon Eclipse E600 fluorescence microscope equipped with 100 Watt mercury lamp, an episcopic-fluorescence attachment, an H-III photomicrographic attachment and various color filters.
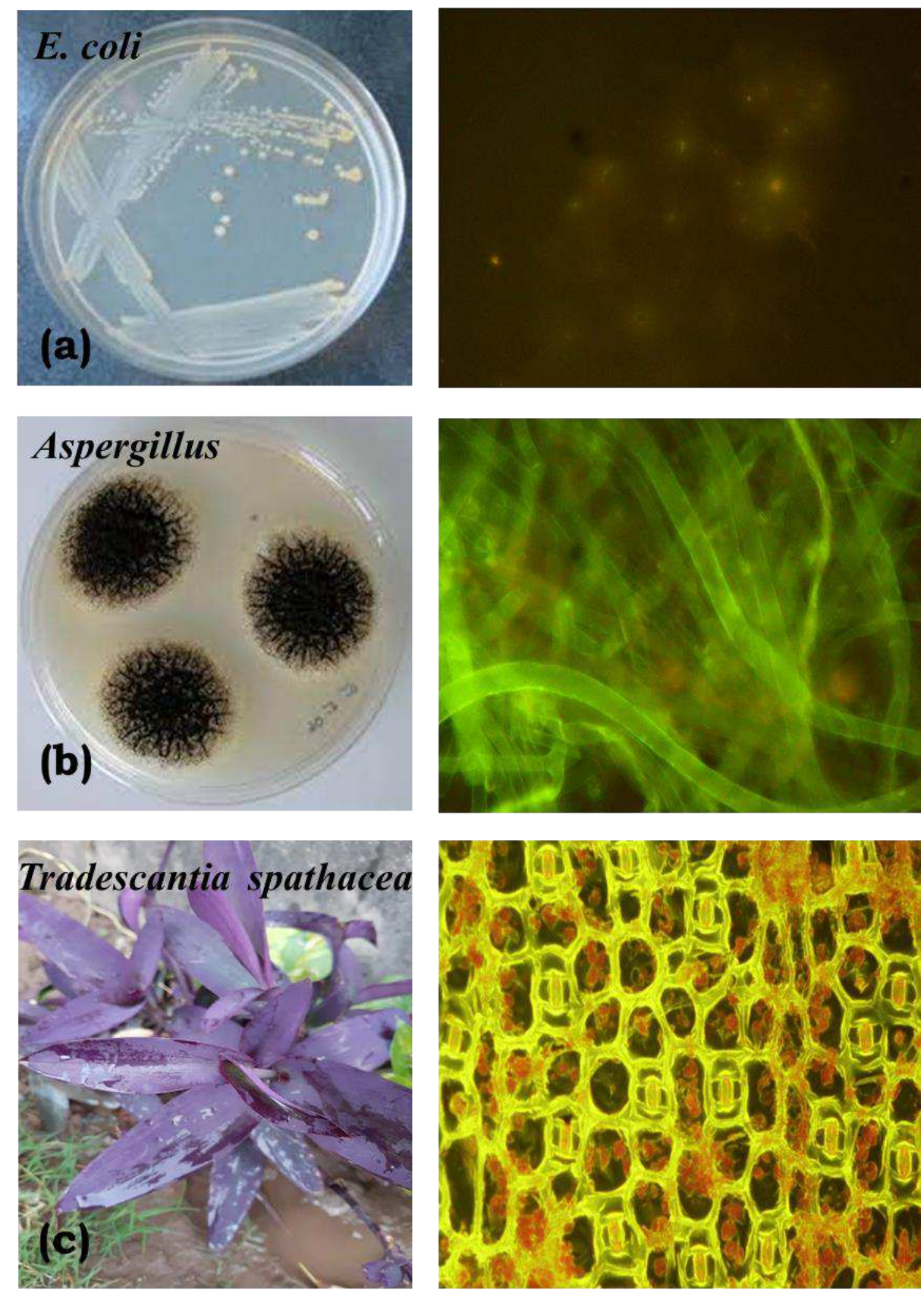

Figure 8: Fluorescent staining of (A) Bacteria (Escherichia coli), (B) fungi (Aspergillus) and (C) Tradescantia spathacea (Sitara) Plant.

Fluorescent microscope was used to analyse the bacterial form and cell configurations, as illustrated in Fig. 8a, which displays a rod-shaped Bacillus and a tiny rod of E coli. Similarly, fungal slides were examined under a fluorescent microscope to determine the type of hyphae, conidiophore, and conidia, as well as their arrangements. The fungal cytoplasm is visible on the slide as a florescent colour region containing hyphae, conidiophores, phialides, and conidia surrounded by fluorescent colour (see Fig. 8b). Figure 8c shows the plant cell wall of tradescantia spathacea, which revealed 
detailed cell structure and stomata. However, it is worth noting that the high emission quantum yield of carbon quantum dots is still remains a challenge and is required for their usage in bioimaging applications. Furthermore, the defects in carbon quantum dots play an essential role in the fluorescence nature, and bioimaging sensitivity or selectivity must be enhanced.

\section{Conclusions}

In conclusion,, utilising a microwave assisted synthesis approach, carbon quantum dots were synthesised from the medicinal plant Calotropis gigantea as a green source in a one-step, easy, ecofriendly, and cost-effective manner. Following UV-excitation, the as-synthesized carbon dots demonstrated excellent fluorescence intensity, high photostability, and efficient multicolor fluorescent emission. In fact, as-synthesized CQD fluorescence quantum yields (QYs) achieved 4.24 percent. According to HRTEM and XRD studies, CQDs have an average size of less than $10 \mathrm{~nm}$, a near-spherical form, and are mainly crystalline in nature. Meanwhile, NMR spectra showed the existence of both $\mathrm{sp}^{2}$ and $\mathrm{sp}^{3}$ carbon groups in significant amounts. Furthermore, the as-synthesized CQDs were found to be incipiently instable in water suspension, with Zeta potential measurements revealing the presence of a negative surface charge $(-13.8 \mathrm{mV})$. Because of their simplicity, cheap cost, and green production, CQDs produced from Calotropis gigantea have been standardised as an alternative fluorescent staining agent in biolabeling of bacteria, fungus, and plant cells.

\section{Declarations}

Funding: Not applicable.

Conflicts of interest: The authors declare no conflicts of interest.

Availability of data and material: Not applicable.

Code availability: Not applicable.

Ethics approval: Not applicable.

Consent to participate: Not applicable.

Consent for publication: Not applicable.

Acknowledgments: The authors declare no acknowledgement. 


\section{References}

1. B. Peixotode and O. Monteiroda, Mater Lett. 282, 128764 (2021).

2. D. Ghosh, K. Sarkar, P. Devi, K.-H. Kim, and P. Kumar, Renew. Sust. Energ. Rev. 135, 110391 (2021).

3. Q. Xu, J. Gao, S. Wang, Y. Wang, D. Liu, and J. Wang, J. Mater. Chem. B. 9, 5765 (2021). 4. Y. Wei, L. Chen, S. Zhao, X. Liu, Y. Yang, J. Du, Q. Li, and S. Yu, Front. Mater. Sci. 15, 253 (2021).

5. M. Pajewska-Szmyt, B. Buszewski, and R. Gadzała-Kopciuch, Mater Chem. Phys. 242, 122484 (2020).

6. K. Raji, V. Ramanan, and P. Ramamurthy, New J. Chem. 43, 11710 (2019).

7. X. Y. Xu, R. Ray, Y. L. Gu, H. J. Ploehn, L. Gearheart, K. Raker, and E. Al, J. Am. Chem. Soc. 126, 40 (2004).

8. C. Cheng, M. Xing, and Q. Wu, J. Alloy. Compd. 790, 221 (2019).

9. P. K. Yadav, V. K. Singh, S. Chandra, D. Bano, V. Kumar, M. Talat, and S. H. H. Hasan, ACS Biomater. Sci. Eng. 5, 623 (2019).

10. C. Cheng, M. Xing, and Q. Wu, Opt. Mater 88, 353 (2019).

11. C. Cheng, M. Xing, and Q. Wu, Mater. Sci. Eng. C 99, 611 (2019).

12. S. Chernyak, A. Podgornova, S. Dorofeev, S. Maksimov, K. Maslakov, S. Savilov, and V. Lunin, , Appl. Surf. Sci. 507, 145027 (2020).

13. J. Xu, L. Dai, C. Zhang, Y. Gui, L. Yuan, Y. Lei, and B. Fan, Bioresour. Technol. 305, 123043 (2020).

14. R. Atchudan, T. N. J. I. Edison, K. . Aseer, S. Perumal, N. Karthik, and L. Y.R., Biosens. Bioelectron. 99, 303 (2018).

15. F. Cui, Y. Ye, J. Ping, and X. Sun, Biosens. Bioelectron. 156, 112085 (2020).

16. A. Abbas, L. T. Mariana, and A. N. Phan, Carbon N. Y. 140, 77 (2018).

17. N. Mahat and S. Shamsudin, Diam. Relat. Mater. 102, 107660 (2020).

18. G. Liu, B. Li, Y. Liu, Y. Feng, D. Jia, and Y. Zhou, Appl. Surf. Sci. 487, 1167 (2019).

19. J. Li, K. Tang, J. Yu, H. Wang, M. Tu, and X. Wang, R. Soc. Open Sci. 6, 181557 (2019).

20. S. Xue, Y. Yang, Y. Sun, J. Fan, X. Li, and Z. Zhang, Int. J. Biol. Macromol. 122, 954 (2019).

21. S. and Atabaev, Nanomater. 8, 342 (2018).

22. A. Raji, E. Thomas, P. Suguna, and V. Rajangam, J. Mol. Liq. 296, 111817 (2019).

23. V. Mehta, S. Jha, H. Basu, R. Singhal, and S. Kailasa, Sensor. Actuator. B Chem. 213, 434 (2015).

24. R. Atchudan, T. Edison, S. Perumal, N. Muthuchamy, and Y. Lee, J. Photochem. Photobiol. Chem. 390, 112 (2020).

25. Z. Xinyue, J. Mingyue, N. Na, C. Zhijun, L. Shujun, L. Shouxin, and L. Jian, Chem. Sus. Chem 11, 11 (2018).

26. L. Sen, T. Jingqi, W. Lei, Z. Yingwei, Q. Xiaoyun, L. Yonglan, A. Abdullah, A. Abdulrahman, and S. Xuping, Adv. Mater 24, 2037 (2012).

27. M. Reza, B. Samaneh, I. Siavash, and V. Rajender, Green Chem. 18, 20 (2016).

28. S. Swagatika, B. Birendra, M. Tapas, and M. Sasmita, Chem. Commun. 48, 8835 (2012).

29. W. Jumeng, L. Bitao, and Y. Peng, RSC Adv. 4, 63414 (2014).

30. S. Jie, S. Shaoming, C. Xiuying, W. Dan, and C. Yan, Mater. Sci. Eng., C 76, 856-864 (2017). 31. W. Jing, W. Cai-Feng, and C. Su, Angew. Chem. 51, 9297-9301 (2012).

32. Y. Bangda, D. Jianhui, P. Xue, L. Qian, Z. Jiangna, L. Qiujun, C. Qiong, L. Haitao, T. Hao, Z. Youyu, and Y. Shouzhuo, Analyst 138, 6551-6557 (2013).

33. S. Abhay and P. Gopinath, Analyst 140, 4260 (2015).

34. G. Dan, S. Shanoming, Y. Qin, and J. Shen, Appl. Surf. Sci. 390, 38 (2016).

35. A. Chatterjee and S. Pakrashi, V-IV. New Delhi Natl. Inst. Sci. Commun. Inf. Resour. 128 (2003).

36. M. Alhede, C. Stavnsbjerg, and T. Bjarnsholt, J. Pathol. Microbiol. Immunol. 126, 779 (2018). 37. H. Ao, H. F. . Pan, Z. Bao, Z. Li, J. Chen, and Z. Qian, ACS Appl. Nano Mater 1, 5673 (2018). 
38. E. Hutter and D. Maysinger, Nano-Bio-Imaging Anal. 74, 592 (2010).

39. S. Thangudu, P. Kalluru, and R. Vankayala, Bioengineering 7, 20 (2020).

40. L. Ying, D. Warren, and H. Olli, BiolFertil Soils 39, 301 (2004).

41. M. . Romero, F. Alves, M. D. Stringasci, H. . Buzza, H. Ciol, N. M. Inada, and V. S. Bagnato, Front. Microbiol. 12, 662149 (2021).

42. A. Peter, P. Julio, and K. James, Atkins' Physical Chemistry (Oxford University Press, 2018).

43. L. Zhenxiang, G. Weina, and L. Chang, J. Vet. Med. Sci. 80, 427 (2018).

44. H. Deng, L. X. Yin-Huan, L. Ke-Lin, Z. Qiong-Qiong, P. Hua-Ping, L. Ai-Lin, O. Xing-Hua, and L. Wei, Nanoscale 0, 10292-10300 (2017).

45. D. Cullity, Elements of X-Ray Diffraction (Pearson, 2001).

46. W. Weiping, L. Ya-Chun, H. Hong, F. Jiu-Ju, C. Jian-Rong, and W. Ai-Jun, Analyst 139, 1692-1696 (2014).

47. H. Pin-Che, S. Zih-Yu, L. Chia-Hsin, and C. Huan-Tsung, Green Chem. 14, 917 (2012). 48. S. Hanjun, Z. Andong, G. Nan, L. Kai, R. Jinsong, and Q. Xiaogang, Angew. Chem. 54, 7176-7180 (2015).

49. K. Mária, M. Zoran, H. Petr, M. Matej, Š. Helena, A. K., M. D., K. Pavel, V. Jan, C. Zdenka, L. Marián, M. Lukaš, M. Biljana, and Š. Zdeno, ACS Biomater. Sci. Eng. 4, 3983 (2018).

50. R. A. Hoffman, S. Forsen, and B. Gestblom, Analysis of NMR Spectra (, Springer-Verlag Berlin Heidelberg, 1971).

51. X. . Jia, J. Li, and E. Wang, Nanoscale 4, 5572 (2012).

52. I. Siavash and V. Rajender, Environ. Chem. Lett. 18, 1 (2020).

53. L. Bányai and W. Koch, Semiconductor Quantum Dots (World Scientific, Singapore, n.d.). 54. M. E. Fuller, S. H. Streger, R. K. Rothmel, B. J. Mailloux, J. A. Hall, T. C. Onstott, J. K. Fredrickson, D. L. Balkwill, and M. F. DeFlaun, Environ. Appl. Environ. Microbiol. 66, 4486 (2000). 